\title{
Hepatoprotective effects of remote perconditioning during renal ischemia
}

\author{
Sedaghat $\mathrm{Z}^{1}$, Kadkhodaee $\mathrm{M}^{2}$, Seifi $\mathrm{B}^{2}$, Ahghari $\mathrm{P}^{3}$ \\ Department of Physiology and Pharmacology, School of Medicine, Bushehr University of Medical Sciences, Bushehr, Iran. \\ kadkhodm@tums.ac.ir
}

\begin{abstract}
Background: Novel treatment strategies are required to reduce the development of hepatic injury during surgical procedure in which renal ischemia/reperfusion (IR) is inevitable. Remote perconditioning ( $\mathrm{rPeC})$ has been proved to reduce the extent of kidney damages induced by renal IR injury. The aim of this study was to determine the protective effect of $\mathrm{rPeC}$ against hepatic injury caused by renal ischemia.

Methods: Male rats were subjected to the right nephrectomy and randomized as: sham, no additional intervention; IR, 45 min of left renal pedicle occlusion; $\mathrm{rPeC}$, four cycles of 5-min limb IR administered at the beginning of renal ischemia. After 24-h of reperfusion, the plasma and tissue samples were taken.

Results: A significant improvement in hepatic functional injury and oxidative damages were observed in the $\mathrm{rPeC}$ group compared to the IR group. However, histological evaluation and plasma levels of TNF- $\alpha$ revealed no significant difference among groups.

Conclusions: It is concluded that $\mathrm{rPeC}$ exerted protective effects on renal IR-induced hepatic injury as a remote organ. The protection may be a consequence of the reduction in oxidative stress in the liver. This simple approach may be a promising strategy against IR-induced remote organ damages in the clinical practice (Fig. 4, Ref. 23). Text in PDF www.elis.sk.

Key words: hepatic, kidney, oxidative stress, remote organ.
\end{abstract}

Remote organ injury is a kind of oxidative damage that may develop in various organs away from the main tissue exposed to ischemia/reperfusion (IR). IR injury (IRI) is an unavoidable process, which occurs during surgical procedure such as renal transplantation and nephron-sparing surgeries. It results in severe local and systemic injury, which contributes to an increased morbidity and mortality in these operations. Since multiorgan failure is a widespread consequence of severe renal injury, hepatic damage as a result of renal IR seems to be an extensive encountered problem (1).

IRI involves multifactorial mechanisms and distinct metabolic pathways leading to severe single organ injury or multiorgan failure and death. IR damage induces an inflammatory response, which results in the formation of reactive oxygen species (ROS) that augments local tissue injury or affects organs remote from the site of IR. An important function of ROS is the regulation of cytokine expression (2). Novel powerful treatment strategies are required to reduce the development of kidney and other vital organ injuries. These protections may reduce the magnitude of IR

${ }^{1}$ Department of Physiology and Pharmacology, School of Medicine, Bushehr University of Medical Sciences, Bushehr, Iran, ${ }^{2}$ Department of Physiology, School of Medicine, Tehran University of Medical Sciences, Tehran, Iran, and ${ }^{3}$ Department of Physiology, School of Medicine, Tehran University of Medical Sciences, International Campus (TUMS-IC), Tehran, Iran

Address for correspondence: M. Kadkhodaee, Department of Physiology, School of Medicine, Tehran University of Medical Sciences, Tehran 14176-13151, Iran.

Phone: +982188259862, Fax: +982166419484 injury and improve postoperative outcomes. Among these strategies, remote ischemic conditioning represents a feasible and reliable tool. This technique is based on the fact that short intermittent IR episodes of remote non-vital organs such as limb before (preconditioning, PC), after (postconditioning, POC) or during (perconditioning, $\mathrm{rPeC}$ ) prolonged lethal ischemic period provides protection against IRI (3).

The degree of systemic and local damages resulting from the IR depends on the duration of the ischemia. It is important to notice that some detrimental effects of ischemia begin during ischemia and before reperfusion, such as ion channel dysfunctions (4) and caspase activation (5). Thus, induction of protective strategies during ischemia may confer more protection especially in the prolonged ischemic periods, which occur during surgical procedures. $\mathrm{rPeC}$ is a novel protective strategy, in which short-time intermittent IR of a remote organ, right at the beginning of prolonged IR, protects the main ischemic tissue from the injury. This method was first described in the heart in 2007 (6). The renoprotective effect of $\mathrm{rPeC}$ was for the first time reported in 2011 (7). The present study was designed to investigate whether $\mathrm{rPeC}$ is able to exert systemic effect and decrease liver injury after renal IR.

\section{Methods}

Before the study, all protocols were approved by the institution's animal welfare regulatory committee, and/or all protocols were in conformity with the Guide for the Care and Use of Laboratory Animals published by the National Institutes of Health 86-23, 
675-679

revised in 1985. Male Sprague-Dawley rats, weighting 220-270 g, were housed under standard conditions (12 hours light/day cycle and environment temperature of $20-22^{\circ} \mathrm{C}$ ) and had free access to water and standard laboratory food.

\section{Surgical procedures}

Animals were anesthetized with intraperitoneal injection of sodium pentobarbital ( $60 \mathrm{mg} / \mathrm{kg}$ body wt). Additional doses (12 $\mathrm{mg} / \mathrm{kg}$ body $\mathrm{wt}$ ) were administered as needed. Systolic blood pressure and heart rate were monitored by a tail cuff connected to a pulse transducer device (MLT125/R, ADInstruments). The transducer was connected to a PowerLab/4SP data acquisition system (software Chart, version 5, ADInstruments, Castle Hill, Australia).

After right nephrectomy through a flank incision, rats were subjected to the following protocols ( $\mathrm{n}=6$ in each group):

Control group (sham): Animals were subjected to the isolation of left renal pedicle without ligation;

Ischemia reperfusion group (IR): Animals were subjected to 45 min of left renal pedicle occlusion by a nontraumatic microvascular clamp;

Remote perconditioning group ( $\mathrm{rPeC})$ : Animals were subjected to $45 \mathrm{~min}$ of left renal pedicle occlusion, during which 4 cycles of 5 min of intermittent ischemia and reperfusion of the left femoral artery applied just at the beginning of renal ischemia.

After 24 hours of reperfusion, animals were anaesthetized and blood samples were collected from inferior vena cava. Liver tissue samples were harvested from the same lobe and washed in the cold phosphate-buffered saline. A piece of tissue were fixed in $10 \%$ formalin for histological processing and the rest were immediately snap-frozen with liquid nitrogen and stored at $-70{ }^{\circ} \mathrm{C}$ until further studies.

\section{Functional assessments}

Kidney (BUN \& creatinine) and liver (ALT, AST, and LDH) functional indices were assessed in plasma by the colorimetric methods using Hitachi 704 autoanalyzer, Japan.

\section{Oxidative stress assessments}

Measurement of malondialdehyde (MDA) levels: MDA levels were measured in the liver tissue samples according to Esterbauer and Cheeseman method (8). MDA reacts with thiobarbituric acid producing a pink pigment that has a maximum absorption at $532 \mathrm{~nm}$.

Measurement of superoxide dismutase (SOD) activity: SOD activity was measured in the liver tissue according to the Paoletti et al method (9). In this assay, superoxide anion is generated from $\mathrm{O}_{2}$ in the presence of ethylenediaminetetraacetic acid (EDTA), mercaptoethanol and manganese (II) chloride. Oxidation of nicotinamide adenine dinucleotide phosphate (NADPH) is linked to the availability of superoxide anions in the medium.

\section{Plasma TNF- $\alpha$ assessment}

Plasma TNF- $\alpha$ levels were determined using an enzyme-linked immunosorbent assay kit (BioLegend's Rat TNF- $\alpha$ LEGEND MAX $^{\mathrm{TM}}$ ELISA kit; Biolegend, CA, USA).

\section{Histological assessment}

After 24 hours of reperfusion, liver tissues were harvested and immediately fixed in $10 \%$ formalin. The fixed tissues were then embedded in paraffin, cut into $4-\mu \mathrm{m}$-thick sections, deparaffinized, dehydrated and stained with haematoxylin and eosin. The sections were examined under a light microscope for the presence of congestion, cellular degenerative changes and cytoplasmic vacuolization.

\section{Statistical analysis}

All results are expressed as the mean \pm SEM. One-way ANOVA was used to compare mean values across groups followed by Tukey test. $\mathrm{p}<0.05$ was considered significant.

\section{Results}

Effect of $r P e C$ on the liver functional indices

Acute kidney injury was confirmed by a significant increase in plasma BUN and creatinine $(117 \pm 4.4 \mathrm{mg} / \mathrm{dl}$ and $4.02 \pm 0.20$ $\mathrm{mg} / \mathrm{dl}$ respectively; $\mathrm{p}<0.001 \mathrm{vs}$ sham) in IR group compared to the sham-operated rats $(20.8 \pm 0.55 \mathrm{mg} / \mathrm{dl}$ and $0.64 \pm 0.05 \mathrm{mg} / \mathrm{dl})$. This is in accordance with our previous finding, which have been recently published (7). After 24 hours of renal reperfusion, plasma levels of ALT $(142.2 \pm 13.0$ vs $86.8 \pm 9.8 \mathrm{U} / \mathrm{L}, \mathrm{p}<0.05)$, AST ( $455.8 \pm 38.7$ vs $203.3 \pm 19.9 \mathrm{U} / \mathrm{L}, \mathrm{p}<0.001)$, and LDH (3816.3 \pm 1357.9 vs $335.5 \pm 75.45 \mathrm{U} / \mathrm{L}, \mathrm{p}<0.05$ ) were significantly increased in the renal IR group compared to the sham group. $\mathrm{rPeC}$ significantly attenuated ALT ( $78 \pm 8.1 \mathrm{U} / \mathrm{L}, \mathrm{p}<0.05)$, AST $(216.3$ $\pm 21.8 \mathrm{U} / \mathrm{L}, \mathrm{p}<0.001)$ and $\mathrm{LDH}(338.2 \pm 90.0 \mathrm{U} / \mathrm{L}, \mathrm{p}<0.05)$ in the plasma compared to the renal IR group (Fig. 1).

\section{Effect of $r P e C$ on the liver oxidative stress markers}

MDA levels: Renal ischemia/reperfusion resulted in a significant increase in the hepatic tissue MDA contents $(0.76 \pm 0.06$ vs $0.07 \pm 0.04 \mu \mathrm{mol} / 100 \mathrm{mg}$ tissue, $\mathrm{p}<0.05)$ compared to the sham group. $\mathrm{rPeC}$ significantly decreased MDA contents $(0.23$ $\pm 0.08 \mu \mathrm{mol} / 100 \mathrm{mg}$ tissue, $\mathrm{p}<0.05)$ compared to the renal IR group (Fig. 2a).

SOD activity: Renal ischemia/reperfusion resulted in a significant decrease in the hepatic tissue SOD activity $(10.11 \pm 2.62$ vs $30.87 \pm 1.13 \mathrm{U} / \mathrm{g}$ tissue, $\mathrm{p}<0.05$ ) compared to the sham group. $\mathrm{rPeC}$ significantly increased SOD activity $(22.11 \pm 2.15 \mathrm{U} / \mathrm{g}$ tissue, $\mathrm{p}<0.05$ ) compared to the IR group (Fig. 2b).

\section{Effect of $r P e C$ on the plasma levels of TNF- $\alpha$}

After 24 hours of reperfusion, there was no significant differences $($ sham $=11.2 \pm 2.4, \mathrm{IR}=11.5 \pm 1.0$ and $\mathrm{rPeC}=10.3 \pm$ $1.5 \mathrm{pg} / \mathrm{ml}$ ) among groups in the plasma levels of TNF- $\alpha$ (Fig. 3).

\section{Effect of rPeC on the liver histology}

Livers from sham-operated rats almost had a normal histology. After 24 hours of reperfusion, the levels of tissue injury in renal IR group was minimal. Although there were enlarged sinusoidal space and scattered areas of nuclear condensation, but no sign of tissue congestion or nuclear and cytoplasm degenerative changes 

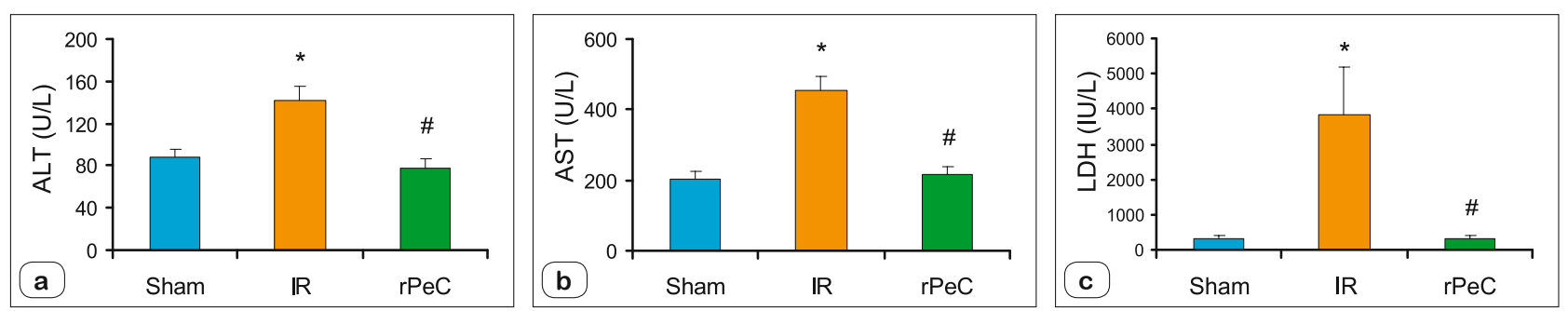

Fig. 1. Effect of rPeC on plasma levels of ALT (a), AST (b) and LDH (c). IR - ischaemia/reperfusion; rPeC - remote perconditioning. Data are expressed as the mean $\pm \operatorname{SEM}\left(n=6\right.$ in each group). ${ }^{*} \mathrm{P}<0.05$ vs sham group, $\# \mathrm{P}<0.05$ vs IR group.

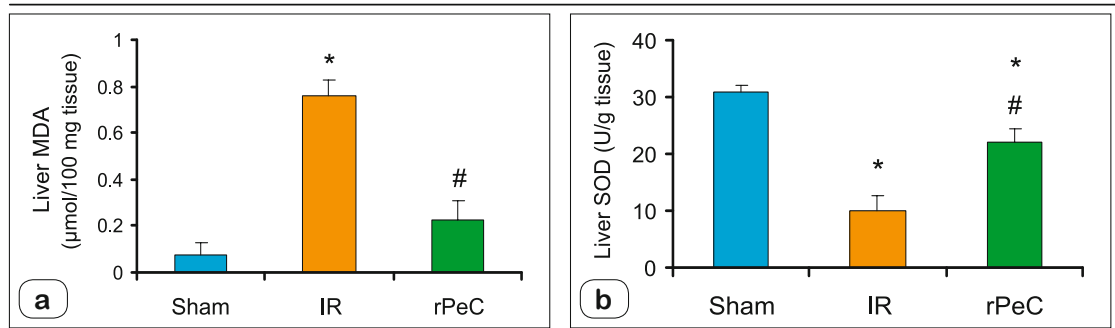

Fig. 2. Effect of rPeC on MDA levels (a) and SOD activities (b) in the liver. IR - ischaemia/ reperfusion; $\mathrm{rPeC}-$ remote perconditioning. Data are expressed as the mean $\pm \mathrm{SEM}(\mathrm{n}=6$ in each group). * $\mathrm{P}<0.05$ vs sham group, \# $\mathrm{P}<0.05$ vs IR group.

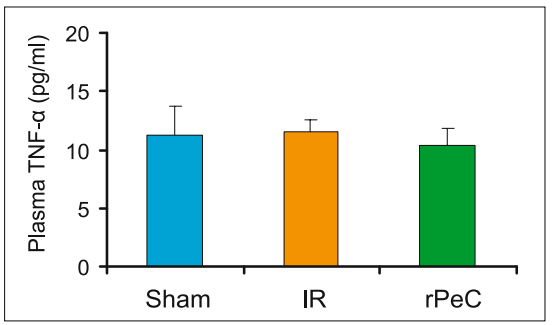

Fig. 3. Plasma levels of TNF- $\alpha$ (c). IR - ischaemia/reperfusion; rPeC - remote perconditioning. Data are expressed as the mean \pm SEM $(n=6$ in each group).
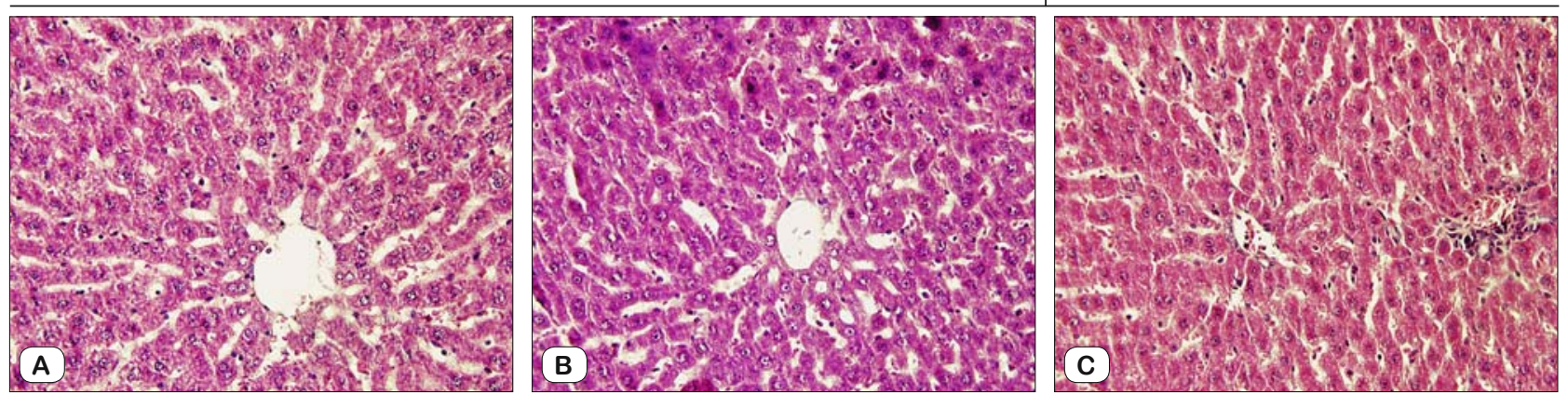

Fig. 4. Liver histology in experimental groups. Sham operation (A), ischemia reperfusion (B) and remote perconditioning (C). Livers from sham-operated rats had normal histology. After 24 hours of reperfusion, the levels of tissue injury in liver IR group was minimal. Although there were enlarged sinusoidal space and scattered areas of nuclear condensation, no sign of tissue congestion or cytoplasm degenerative changes were seen. Tissues of $\mathrm{rPeC}$ group showed a normal histology comparable with sham group. Infiltration of leukocytes in both IR and $\mathrm{rPeC}$ was present. Magn. $\mathrm{x} 400$ (A-C).

were seen. Tissues of rPeC group showed a normal histology comparable with the sham group. Infiltration of leukocytes in both IR and $\mathrm{rPeC}$ was present (Fig. 4).

\section{Discussion}

Results of this study demonstrated a novel strategy against renal IR-induced hepatic injury. Data from our laboratory showed that $\mathrm{rPeC}$, in addition to the protection of ischemic kidneys, prevented hepatic functional impairment demonstrated by decreases in ALT, AST and LDH levels in the plasma. Moreover, $\mathrm{rPeC}$ had the ameliorating effects on oxidative stress in the liver by a significantly lower MDA level and significantly higher SOD activity compared to non-conditioned rats. However, at the time of evaluation (24 hours after reperfusion) we did not detect any significant difference in plasma levels of TNF- $\alpha$ and histological parameters among groups.
Renal IRI occurs in many clinical situations like transplantation, partial nephrectomy, or elective urologic operations. Many studies have reported injuries to other organs, including liver injury during the course of renal IR. In this study, 45 minutes of renal ischemia and 24 hours of reperfusion resulted in liver damage, which was characterized by significantly increased serum transaminases and LDH levels. The release of AST and ALT and LDH into the plasma reflected mostly the damages to hepatocyte. These results are in accordance with previous studies, showing that renal ischemia remotely causes detrimental changes in the function of liver $(1,10)$.

Remote perconditioning was recently reported to protect the kidney from renal IRI. Its protective effect was associated with attenuated oxidative stress damages in the local ischemic organ, namely the kidneys (11). However, the remote protective effect of $\mathrm{rPeC}$ was yet not reported. Thus, in the present study, we evaluated the effect of $\mathrm{rPeC}$ on the liver as a distant organ after renal 
ischemia. There are some reports in recent years on the systemic effect of ischemic conditioning. Saxena et al demonstrated that ischemic PC provokes a systemic protective response in the body. The microarray analysis of blood samples from healthy human volunteers indicated that forearm PC suppressed proteins that are involved in leukocyte exocytosis and implicated in IRI (12). In a study by Peralta et al the remote protective effect of PC was demonstrated (13). They showed that local liver ischemic preconditioning abrogated the increase in P-selectin up-regulation, prevented neutrophil infiltration and thus reduced the oxidative stress and microvascular disorders in the remote organ, namely the lungs, following hepatic IRI.

Reactive oxygen species (ROS) are among the most important factors generated by ischemic tissues and activated neutrophils after renal reperfusion (14). Attracted and activated neutrophils migrate and adhere to endothelium, causing the release of proteases and oxygen free radicals, which may trigger the activation of liver Kupffer cells. This may lead to hepatic dysfunction. To discard toxic ROS, cells are equipped with antioxidant enzymes such as superoxide dismutase. Earlier reports from other investigators demonstrated significantly lower SOD activities in the local ischemic organ, which was improved using ischemic conditioning therapy (15). In the present study, SOD activity was found to be significantly lower in the remote organ, liver, of IR group compared to the sham. This is in accordance with previous studies that reported renal IRI was linked with decreased SOD activity in the liver tissues. Recently, the study by Khastar et al showed that transfusion of isolated leukocytes from mice that underwent renal IR, caused liver functional impairment and hepatic oxidative injury in the intact recipient mice (16). The present study indicated that $\mathrm{rPeC}$ prevented renal IR-induced SOD depletion in the liver. We have recently observed a decreased renal SOD activity in an acute kidney injury model (11). The local protective effects of different methods of ischemic conditioning through increases in SnOD activity have reported by Yun et al (17) and Kim et al (18) in renal IR models.

Tissue MDA content is a reliable index of lipid peroxidation. Previous studies have provided evidence indicating implication of MDA in renal IR-induced liver injury (19). In the present study, we demonstrated that renal IR resulted in increased MDA content in hepatic tissues and it was associated with impaired liver function. $\mathrm{rPeC}$ prevented renal IR-induced increases in liver tissue MDA levels.

In this study, we did not observe any significant difference in the plasma levels of TNF- $\alpha$ among groups. Some aspects of IRI like inflammatory gene responses occur in a time dependent pattern. The results of a randomized controlled trial evaluating the effects of remote ischemic preconditioning on children undergoing cardiac surgery indicated that inflammatory responses after IR was significant in the acute phase of reperfusion and were markedly attenuated at 24 hours. They observed that the changes in plasma levels of TNF- $\alpha$ after IR, indicated a time dependent pattern which significantly increased after 3 and 6 hours after operation and returned to the preoperation levels after 24 hours (20). Furthermore, in vivo dog experiments have demonstrated a time-dependent myocardial dysfunction following intravenous infusion of TNF- $\alpha$ (21).

\section{Clinical applications}

It is noteworthy that $\mathrm{rPeC}$ is not only an object of academic interest, but has been applied in clinical trials in the heart operations. In a clinical trial, Li et al reported a reduction in myocardial injury over cardioplegia in adults undergoing selective valve replacement (22). Recently, Hougaard et al showed the feasibility of $\mathrm{rPeC}$ in acute stroke patients (23).

\section{Conclusion}

In the present study, remote perconditioning exerted protective effects on acute kidney injury-induced hepatic damages as a remote organ. In this regard, remote perconditioning may offer a novel noninvasive and cost-free treatment strategy for decreasing the incidence of liver injury in patients undergoing renal transplantation or other clinical situations in which renal ischemia reperfusion is inevitable. This protection may be a consequence of the reduction in oxidative stress in the liver.

\section{References}

1. Wang B, Bai M, Bai Y, Li Q. Liver injury following renal ischemia reperfusion in rats. Transplant Proc 2010; 42: 3422-3426.

2. Remick DG, Villarete L. Regulation of cytokine gene expression by reactive oxygen and reactive nitrogen intermediates. J Leukoc Biol 1996; 59: 471-475.

3. Hausenloy DJ, Yellon DM. The therapeutic potential of ischemic conditioning: an update. Nat Rev 2011; 8: 619-629.

4. Steenbergen C, Perlman ME, London RE, Murphy E. Mechanism of preconditioning. Ionic alterations. Circ Res 1993; 72: 112-125.

5. Stephanou A, Brar B, Liao Z, Scarabelli T, Knight RA, Latchman DS. Distinct initiator caspases are required for the induction of apoptosis in cardiac myocytes during ischaemia versus reperfusion injury. Cell Death Differ 2001; 8: 434-435.

6. Schmidt MR, Smerup M, Konstantinov IE, Shimizu M, Li J, Cheung M, White PA, Kristiansen SB, Sorensen K, Dzavik V, Redington AN, Kharbanda RK. Intermittent peripheral tissue ischemia during coronary ischemia reduces myocardial infarction through a KATP-dependent mechanism: first demonstration of remote ischemic perconditioning. Am J Physiol Heart Circ Physiol 2007; 292: H1883-1890.

7. Kadkhodaee M, Seifi B, Najafi A, Sedaghat Z. First report of the protective effects of remote per- and postconditioning on ischemia/reperfusioninduced renal injury. Transplantation 2011; 92: 55-56.

8. Esterbauer H, Cheeseman KH. Determination of aldehydic lipid peroxidation products: malonaldehyde and 4-hydroxynonenal. Methods Enzymol 1990; 186: 407-421.

9. Paoletti F, Mocali A. Changes in CuZn-superoxide dismutase during induced differentiation of murine erythroleukemia cells. Cancer Res 1988; 48: 6674-6677.

10. Kadkhodaee M, Golab F, Zahmatkesh M, Ghaznavi R, Hedayati M, Arab HA, Ostad SN, Soleimani M. Effects of different periods of 
renal ischemia on liver as a remote organ. World J Gastroenterol 2009; 15: 1113-1118.

11. Sedaghat Z, Kadkhodaee M, Seifi B, Salehi E, Najafi A, Dargahi L. Remote per-conditioning reduces oxidative stress, down-regulates cyclooxygenase- 2 expression and attenuates ischaemia/reperfusion-induced acute kidney injury. Clin Exp Pharmacol Physiol 2013; 42: 97-114.

12. Saxena P, Newman MA, Shehatha JS, Redington AN, Konstantinov IE. Remote ischemic conditioning: evolution of the concept, mechanisms, and clinical application. J Card Surg 2010; 25: 127-134.

13. Peralta C, Fernandez L, Panes J, Prats N, Sans M, Pique JM, Gelpi E, Rosello-Catafau J. Preconditioning protects against systemic disorders associated with hepatic ischemia-reperfusion through blockade of tumor necrosis factor-induced P-selectin up-regulation in the rat. Hepatology 2001; 33: 100-113.

14. Yoshitomi T, Hirayama A, Nagasaki Y. The ROS scavenging and renal protective effects of $\mathrm{pH}$-responsive nitroxide radical-containing nanoparticles. Biomaterials 2011; 32: 8021-8028.

15. Yong J, Bo Y, Bao-qiang W, Jian-jun T, Zhen Q. The optimal time window of ischemic preconditioning (IPC) on the reperfusion injury in moderate to severe hepatocirrhosis in rats. Ann Clin Lab Sci 2013; 43: 64-9.

16. Khastar H, Kadkhodaee M, Sadeghipour HR, Seifi B, Hadjati J, Delavari F, Soleimani M. Leukocyte involvement in renal reperfusioninduced liver damage. Ren Fail 2011; 33: 79-83.

17. Yun Y, Duan WG, Chen P, Wu HX, Shen ZQ, Qian ZY, Wang DH. Ischemic postconditioning modified renal oxidative stress and lipid peroxidation caused by ischemic reperfusion injury in rats. Transplant Proc 2009; 41: 3597-3602.
18. Kim J, Jang HS, Park KM. Reactive oxygen species generated by renal ischemia and reperfusion trigger protection against subsequent renal ischemia and reperfusion injury in mice. Am J Physiol 2010; 298: F158-166.

19. Gulec B, Coskun K, Yigitler C, Yigit T, Aydin A, Oner K. Ischemiareperfusion injury in the liver during renal transplantation: does perfusion solution play any role? Transplant Proc 2008; 40: 59-62.

20. Cheung MM, Kharbanda RK, Konstantinov IE, Shimizu M, Frndova H, Li J, Holtby HM, Cox PN, Smallhorn JF, Van Arsdell GS, Redington AN. Randomized controlled trial of the effects of remote ischemic preconditioning on children undergoing cardiac surgery: first clinical application in humans. J Am Coll Cardiol 2006; 47: 2277-2282.

21. Walley KR, Hebert PC, Wakai Y, Wilcox PG, Road JD, Cooper DJ. Decrease in left ventricular contractility after tumor necrosis factor-alpha infusion in dogs. J Appl Physiol 1994; 76: 1060-1067.

22. Li L, Luo W, Huang L, Zhang W, Gao Y, Jiang H, Zhang C, Long L, Chen S. Remote perconditioning reduces myocardial injury in adult valve replacement: a randomized controlled trial. J Surg Res 2010; 164: 21-26.

23. Hougaard KD, Hjort N, Zeidler D, Sorensen L, Nørgaard A, Thomsen RB, Jonsdottir K, Mouridsen K, Hansen TM, Cho TH, Nielsen TT, Botker HE, Ostergaard L, Andersen G. Remote ischemic perconditioning in thrombolysed stroke patients: Randomized study of activating endogenous neuroprotection design and MRI measurements. Int J Stroke 2013; 8: 141-146.

Received August 3, 2013. Accepted June 1, 2014. 